\title{
Combined Atriofascicular and Fasciculoventricular Connections
}

\author{
Riko Masaki, MD; Ichiro Watanabe, MD; Toshiaki Kojima, MD; Naohiro Oshikawa, MD; \\ Kimie Okubo, MD; Yasuo Okumura, MD; Hidezou Sugimura, MD; Satoshi Saito, MD; \\ Yukio Ozawa, MD; Katsuo Kanmatsuse, MD
}

\begin{abstract}
A 32-year-old man with a 20-year history of palpitation showed a short PQ interval and delta wave on 12-lead ECG. An electrophysiological study showed that the atrio-His (AH) and His-ventricular intervals were not influenced by rapid right atrial pacing or by bolus injection of adenosine triphosphate (ATP). The AH interval was shortened by coronary sinus ostium (CSos) pacing. Ventricular stimulus to the atrial activation interval was not affected by ATP but shortened by para-Hisian pacing and orthodromic His bundle activation was followed by atrial activation. The tricuspid-inferior vena cava isthmus was blocked by CSos pacing, but Ith conduction existed during low lateral right atrial pacing. (Circ J 2003; 67: 715-717)
\end{abstract}

Key Words: Atriofascicular connection; Cavotricuspid isthmus; Fasciculoventricular connection; Para-Hisian pacing; Wolff-Parkinson-White syndrome

$\mathbf{I}$ n 1937 Mahaim and Benatt reported an accessory pathway connecting the atrioventricular node and ventricle ('Mahaim fiber'), which includes a nodofascicular and a fasciculoventricular connection. The Mahaim fiber has been considered a component of reentrant tachycardia, but recent studies have shown that an atrioventricular connection with slow conduction or an atriofascicular connection constitutes the pathway in atrioventricular reentrant tachycardia and that the nodoventricular and fasciculoventricular connections seldom contribute to this entity? ${ }^{2-7}$ We present a case of a patient who had an atriofascicular connection and a fasciculoventricular connection and also a unidirectional cavotricuspid isthmus block.

\section{Case Report}

A 32-year-old man was referred to hospital because of palpitations and syncope. The 12-lead ECG showed shortening of the PQ interval and delta wave (leads I, II and V6) consistent with preexitation, but the morphology of the first and second QRS complexes in leads $\mathrm{aV}$ and $\mathrm{V}_{1-3}$ was different, suggesting that the degree of preexcitation had changed (Fig 1). Sustained arrhythmia was not recorded on ambulatory ECG nor was arrhythmia induced during a treadmill exercise test.

\section{Electrophysiological Study}

During sinus rhythm, the atrio-His (AH) and His-ventricular (HV) intervals were $73 \mathrm{~ms}$ and $35 \mathrm{~ms}$, respectively, and they did not change significantly during continuous high right atrial pacing at pacing cycle lengths of 1,000 to

(Received December 12, 2001; revised manuscript received February 22, 2002; accepted February 27, 2002)

The Second Department of Medicine, Nihon University School of Medicine, Tokyo, Japan

Mailing address: Ichiro Watanabe, MD, The Second Department of Medicine, Nihon University School of Medicine, 30-1 OyaguchiKamimachi, Itabashi-Ku, Tokyo 173-8610, Japan. E-mail: iwatanab @ med.nihon-u.ac.jp
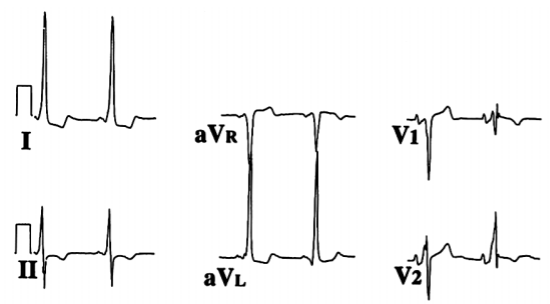

1998 . 6.30
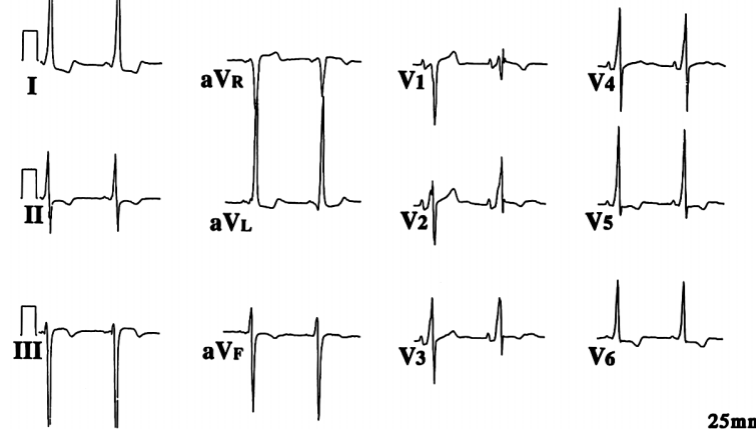

$25 \mathrm{~mm} / \mathrm{s}$

Fig 1. 12-lead ECG showing short PQ interval and delta wave in leads I, II and $\mathrm{V}_{6}$. Note that the PQ interval of the second beat is longer than that of the first beats in leads $\mathrm{V}_{1-3}$ and that the QRS complex of the second beat has an incomplete right bundle branch block pattern.
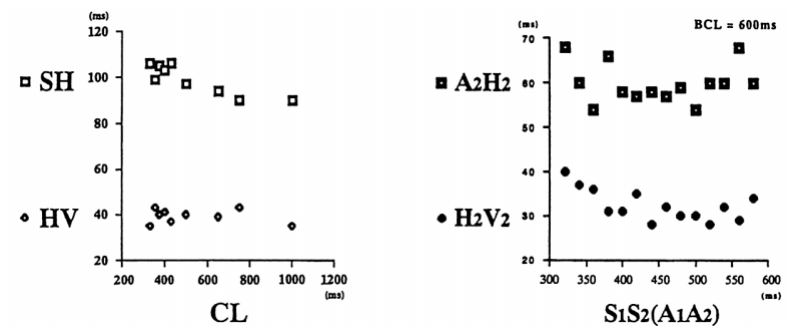

Fig 2. Continuous right atrial pacing (Left) shows a small increase in the stimulus to the His bundle electrogram interval and a constant $\mathrm{HV}$ interval between the pacing cycle lengths of $1,000 \mathrm{~ms}$ and $300 \mathrm{~ms}$. Atrial extrastimuli (Right) have an almost constant $\mathrm{A} 2 \mathrm{H} 2$ interval and a small increase in the H2V2 interval. SH, Stimulus to His bundle electrogram interval; $\mathrm{HV}$, His bundle electrogram to ventricular electrogram interval; CL, cycle length; BCL, basic cycle length; $\mathrm{A} 2 \mathrm{H} 2$, atrio-His bundle electrogram interval in response to atrial extrastimuli. $\mathrm{H} 2 \mathrm{~V} 2$, His bundle electrogram to ventricular electrogram interval in response to atrial extrastimuli. S1S2, coupling interval of the pacing stimuli; A1A2, atrial electrogram interval in response to atrial extrastimuli. 

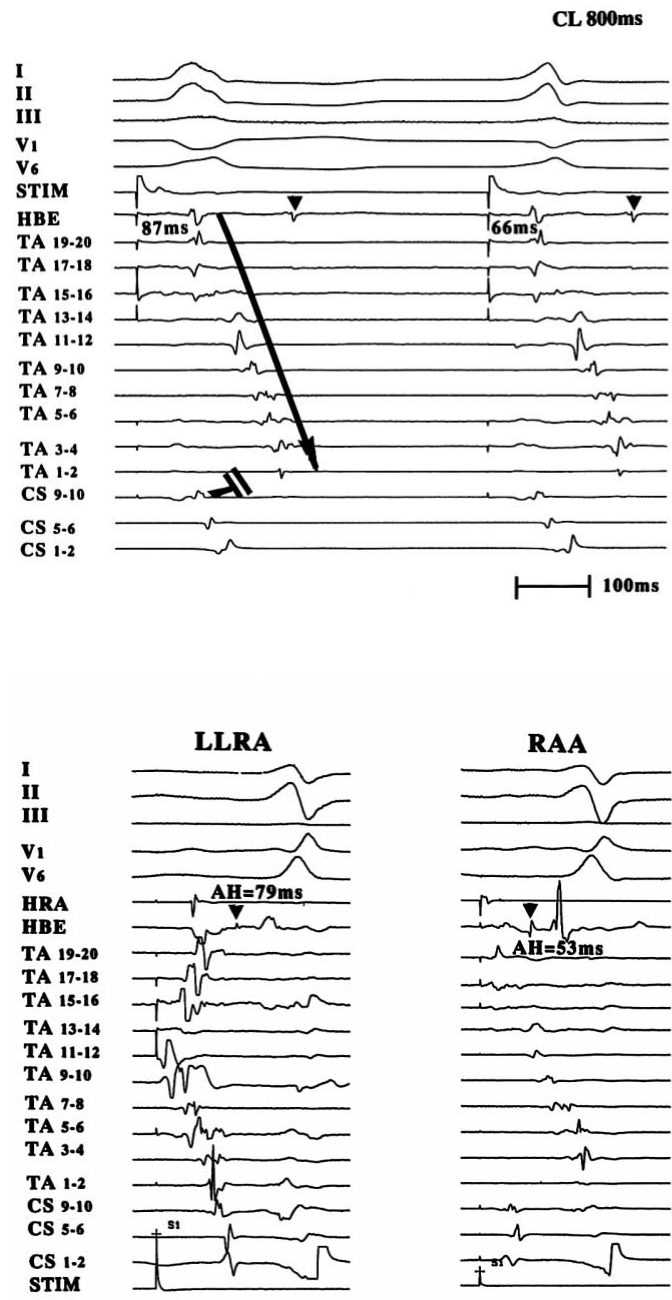
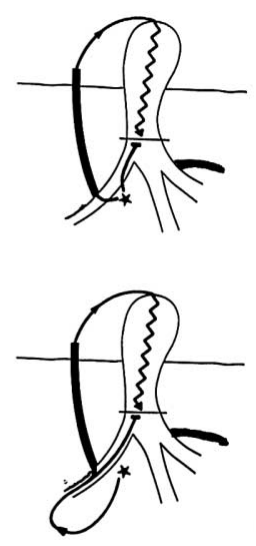

$\longmapsto 100 \mathrm{~ms}$

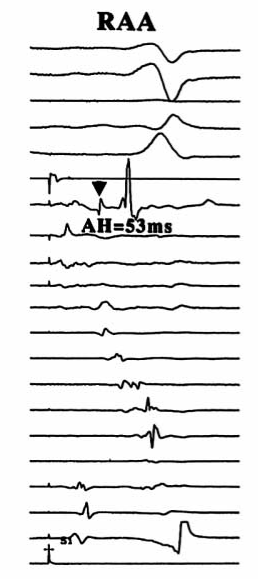

Fig 3. Para-Hisian pacing. The first pacing beat has a wide QRS complex and the stimulus to the A interval on the His bundle electrogram was $87 \mathrm{~ms}$. The second pacing beats has a narrow QRS complex and the stimulus to the A interval on the His bundle electrogram was $66 \mathrm{~ms}$. Thus, in the first beat, only the right ventricle was paced, whereas in the second beat, both the distal His bundle or right bundle and the right ventricle were paced. There was no direct ventriculoatrial connection because the stimulus to the A interval became shorter with His bundle pacing. Orthodromic His bundle activation $(\boldsymbol{\nabla})$ was seen $139 \mathrm{~ms}$ after A in the His bundle electrogram. In addition, the cavotricuspid isthmus was blocked between CS9-10 and TA 1-2. (Right) Schema. ^Right ventricular pacing site.

Fig 4. Comparison of the AH interval in low lateral right atrial pacing (LLRA), right atrial appendage (RAA) pacing and coronary sinus ostium (CSos) pacing. The AH interval was shortest during CSos pacing, suggesting that the atrial end of the atrio-His or atriofascicular fiber arises from the posterior septum. Note that cavotricuspid isthmus conduction exists during LLRA pacing, whereasit was blocked during RAA and CSos pacing. LLRA, low lateral right atrium; RAA, right atrial appendage; CSOS, coronary sinus ostium; CL, pacing cycle length; $\mathbf{\nabla}$, His bundle electrogram; HRA, high right atrium; HBE, His bundle electrogram; TA, tricuspid annulus; CS, coronary sinus; STIM, pacing stimulus; CL, pacing cycle length.

$300 \mathrm{~ms}$ (Fig 2). Atrial extrastimuli at a basic cycle length (BCL) of $600 \mathrm{~ms}$ did not show dual atrioventricular (AV) nodal conduction and the $\mathrm{AH}$ and $\mathrm{HV}$ intervals were also constant until an effective atrial refractory period of $300 \mathrm{~ms}$ (Fig 2). These data suggested that there was not a direct atrioventricular bypass tract. Supraventricular tachycardia was not induced by rapid atrial and ventricular pacing, S1S2 and S1S2S3 atrial and ventricular extrastimuli at BCLs of 600 and $400 \mathrm{~ms}$ at baseline or under isoproterenol infusion $(0.6 \mu \mathrm{g} / \mathrm{min})$. Bolus injection of $20 \mathrm{mg}$ of adenosine triphosphate (ATP) during atrial pacing at a cycle length (CL) of $600 \mathrm{~ms}$ did not affect the $\mathrm{AH}$ and $\mathrm{HV}$ intervals. Polarity and degree of preexcitation were not influenced by pacing from the high right atrium, right atrial appendage (RAA), low lateral right atrium (LLRA) and coronary sinus ostium (CSos). The earliest retrograde atrial activation during right ventricular pacing was recorded at the His bundle electrogram, and the ventriculoatrial (VA) intervals during right ventricular (RV) apex pacing at a CL of $900 \mathrm{~ms}$ and $400 \mathrm{~ms}$ were $122 \mathrm{~ms}$ and $133 \mathrm{~ms}$, respectively. A 2:1 VA block occurred at a pacing CL of $350 \mathrm{~ms}$. Bolus injection of $20 \mathrm{mg}$ of ATP during right ventricular pacing did not affect the VA interval. Because the earliest retrograde atrial activation was seen on the His bundle electrogram during RV pacing at a pacing $\mathrm{CL}$ of $800 \mathrm{~ms}$, we conducted para-Hisian pacing to whether the VA conduction occurred through the His bundle or an accessory atrioventricular pathway? Stim- ulus to the A interval during low voltage pacing was $87 \mathrm{~ms}$, whereas during high voltage pacing it was $66 \mathrm{~ms}$, demonstrating that there was not a direct VA connection on the septum (Fig 3). The His bundle potential was recorded $125 \mathrm{~ms}$ after the atrial electrogram on the His bundle electrogram. We speculated that retrograde VA conduction of the His bundle was blocked at the distal His bundle, but the atrium was activated through retrograde activation of the atriofascicular connection and the atrial activation then conducted to the atrioventricular node and His bundle, but blocked at the distal His bundle. Because the stimulus to the A interval differed during low and high voltage paraHisian pacing, the A-to-second component electrogram interval was the same, showing that the second deflection on the His bundle electrogram was not atrial deflection (Fig 4). In addition, the deflection of the His bundle electrogram during RAA and Csos pacing (Fig 4, middle and right) was $-/+$, but deflection of the second component electrogram on the His bundle electrogram during paraHisian pacing (Fig 3 ) was $+/-$, which suggests the direction of His bundle activation was from the opposite site; that is, His bundle activation during RAA pacing was antidromic, orthodromic during para-Hisian pacing, which shows that retrograde activation of the VA conduction was blocked between the $\mathrm{AH}$ or atriofascicular connection and His bundle electrogram recording site. Thus, the insertion of the $\mathrm{AH}$ or atriofascicular connection should be at or below 
the distal His bundle. Furthermore, a halo catheter placed along the tricuspid valve showed a counterclockwise activation sequence and conduction block between the tricuspid annulus (TA) 1-2 and coronary sinus (CS) 9-10 during Csos pacing, which indicated cavotricuspid isthmus block despite that lack of a history of cardiac surgery or catheter ablation of atrial flutter.

We next compared the activation sequence around the tricuspid valve using a 20-pole halo catheter during RAA, LLRA pacing and CSos pacing at a pacing CL of $600 \mathrm{~ms}$ (Fig 4). The degree and polarity of the delta wave in the surface ECG and the HV interval were similar during the three types of pacing. During LLRA pacing, there was clockwise and counterclockwise activation along the tricuspid valve (Fig 4, left), but during RAA and CSos pacing, only counterclockwise activation was recorded (Fig 4, middle and right). Thus, the conduction properties of the cavotricuspid isthmus showed unidirectional block (ie, clockwise conduction block and counterclockwise conduction). Furthermore, the earliest retrograde activation of the atrium during anteroseptal RV pacing with and without His bundle capture occurred almost simultaneously at the His bundle electrogram and CSos (CS 9-10), which suggested that the atrial attachment of the AH or atriofascicular connection was located between the AV node and coronary sinus ostium. If a posteroseptal atrioventricular connection exists, the atrial activation sequence of the His bundle electrogram and coronary sinus ostium during anteroseptal RV pacing with and without His bundle capture may change because the stimulus to retrograde atrial activation was shortened by $21 \mathrm{~ms}$ during His bundle capture; therefore, retrograde atrial activation of the His bundle electrogram should appear earlier than that of CSos. The AH interval was shortest during Csos pacing and longest during LLRA pacing in this patient, which also supports the location of the $\mathrm{AH}$ or atriofascicular connection in the posteroseptal RA.

The cause of palpitation and syncope in this patient was proved to be vasovagal syncope by tilt table test.

\section{Discussion}

The present patient showed (1) constant stimulus to the $\mathrm{AH}$ interval during right atrial pacing at pacing CLs between 1,000 and $300 \mathrm{~ms}$ without decrement of the conduction property of the interval, (2) constant $\mathrm{A} 2 \mathrm{H} 2$ intervals during atrial extrastimuli, (3) lack of effect of a bolus injection of ATP on the AH interval during atrial pacing, and (4) a similar degree and polarity of preexcitation during RAA, LLRA and CSos pacing, all of which indicate an AH or atriofascicular connection. The AH interval was shortest during Csos pacing because the $\mathrm{AH}$ or atriofascicular connection arises from the posterior septum near the CSos. Furthermore, the patient had a constant HV interval during right atrial pacing at pacing CL between 1,000 and $300 \mathrm{~ms}$ and constant $\mathrm{H} 2 \mathrm{~V} 2$ intervals during atrial extrastimuli in the presence of a delta wave on the 12-lead ECG, which indicate the presence of a fasciculoventricular connection. Thus, we diagnosed a combination of $\mathrm{AH}$ or atriofascicular and fasciculoventricular connections.

The shorter PQ interval in the first beat and the different configuration of the QRS complex of the first and second beat in leads $\mathrm{V}_{1-3}$ (Fig 1) can be explained as follows. Both atriofascicular and fasciculoventricular conduction occurred in the first beat, but in the second beat, atriofascicular conduction was blocked and AV nodal conduction and fasci- culoventricular conduction occurred? Furthermore, there was unidirectional block of the cavotricuspid isthmus; that is, isthmus conduction existed during LLRA pacing, but isthmus conduction was blocked during CSos pacing. Takahashi et al reported that $17 \%$ of patients undergoing ablation of atrial flutter have partial isthmus block ${ }^{10}$ and we also recently reported a patient in whom bidirectional cavotricusid isthmus block was found following cardioversion of sustained atypical atrial flutter ${ }^{11}$ and a case of unidirectional cavotricuspid isthmus in a patient with typical counterclockwise atrial flutter! ${ }^{2}$ However, in the present case, we did not conduct detailed double potential mapping across the isthmus, and we did not evaluate the cavotricuspid conduction under isoproterenol infusion, so we can not rule out the possibility of slow conduction of the isthmus during CSos pacing.

\section{Study Limitations}

We did not record the proximal and distal His bundle potentials and right bundle branch potential simultaneously, so the distal end of the $\mathrm{AH}$ or atriofascicular connection was unclear. We did not conduct detailed antegrade and retrograde mapping of the accessory pathway, so the atrial end of the accessory pathway was also unclear. Furthermore, we did not ablate the accessory pathway, so our data remain speculative.

\section{References}

1. Mahaim I, Benatt A. Nouvelles recherches sur les connexions superieures de la branche gauche du faisceau de His-Tawara avec cloison interventriculaire. Cardiologica 1937; 1: 61-73.

2. Klein GJ, Guiraudon GM, Kerr CR, Sharma AD, Yee R, Szabo T, et al. 'Nodoventricular' accessory pathway: Evidence for a distinct accessory atrioventricular pathway with atrioventricular node-like properties. J Am Coll Cardiol 1998; 11: 1035-1040.

3. Gillette PC, Garson A Jr, Cooley DA, McNamara DG. Prolonged and decremental antegrade conduction properties in right anterior accessory connections: Wide QRS antidromic tachycardia of left bundle branch block pattern without Wolff-Parkinson-White configuration in sinus rhythm. Am Heart J 1982; 103: 66-74.

4. Gallagher JJ, Smith WM, Kasell JH, Benson DW Jr, Sterba R, Grant AO. Role of Mahaim fibers in cardiac arrhythmias in man. Circulation 1981; 64: 176-189.

5. Benditt DG, Epstein ML, Benson DW Jr. Dual accessory nodoventricular pathways: Role in paroxysmal wide QRS reciprocating tachycardia. Pacing Clin Electrophysiol 1983; 6: 577-586.

6. Bardy GH, German LD, Packer DL, Coltorti F, Gallagher JJ. Mechanism of tachycardia using a nodofascicular Mahaim fiber. Am J Cardiol 1984; 54: 1140-1141.

7. Ellenbogen KA, Ramirez NM, Packer DL, O'Callaghan WG, Greer GS, Sintetos AL, et al. Accessory nodoventricular (Mahaim) fibers: A clinical review. Pacing Clin Electrophysiol 1986; 9: 868-884.

8. Hirao K, Otomo K, Wang X, Beckman KJ, McClelland JH, Widman L, et al. Para-Hisian pacing: A new method for differentiating retrograde conduction over an accessory AV pathway from conduction over the AV node. Circulation 1996; 94: 1027-1035.

9. Gallagher JJ. Role of nodoventricular and fasciculoventricular connections in tachyarrhythmias. In: Benditt DG, Benson DW, editors. Cardiac preexcitation syndromes: Origins, evaluation, and treatment. Boston: Martinus Nijhoff Publishing; 1986: 201-232.

10. Takahashi A, Shah DC, Jäis P, Hocini M, Clementy J, Haissaguerre M. Partial cavotricuspid isthmus block before ablation in patients with typical atrial flutter. J Am Coll Cardiol 1999; 33: 1996-2000.

11. Ohkubo K, Watanabe I, Kojima T, Oshikawa N, Sugimura H, Masaki $\mathrm{R}$, et al. Cavotricuspid isthmus block without prior cardiac surgery: Two case reports. J Arrhythmia 2000; 16: 238 (abstract in Japanese).

12. Okumura Y, Watanabe I, Kojima T, Masaki R, Oshikawa N, Yamada $\mathrm{T}$, et al. A patient with clockwise and counterclockwise typical atrial flutter who had clockwise conduction block of the cavotricuspd isthmus block and counterclockwise slow conduction of the isthmus (in Japanese): Successful bidirectional isthmus block by a single point ablation of the isthmus. Clin Card Electrophysiol 2002; 25: in press. 